\title{
Biological Markers of Cognition in Exercise: A Mini Review
}

\author{
Keni Gowsi ${ }^{1, *}$, Gopal Krushna Pal' ${ }^{2}$, Karthick Subramanian ${ }^{3}$
}

\section{Keni Gowsi ${ }^{1, *}$, Gopal Krushna Pal' ${ }^{2}$, Karthick Subramanian ${ }^{3}$}

'Department of Physiology, Jawaharlal Institute of Post Graduate Medical Education and Research, Puducherry, INDIA.

${ }^{2}$ School of Medical Sciences, Pondicherry University, Department of Physiology, Jawaharlal Institute of Post Graduate Medical Education and Research, Puducherry, INDIA. ${ }^{3}$ Department of Psychiatry, Mahatma Gandhi Medical College and Research Institute, Puducherry, INDIA.

\section{Correspondence}

\section{Dr. Keni Gowsi}

Senior Resident, Department of Physiology, JIPMER, Puducherry-605 006, INDIA.

Phone: +91-9626610599

Email: keni_doc@yahoo.in

\section{History}

- Submission Date: 25-08-2019;

- Review completed: 10-10-2019;

- Accepted Date: 21-10-2019.

DOI : 10.5530/ijcep.2019.6.3.22

\section{Copyright}

(c) 2019 Phcog.Net. This is an openaccess article distributed under the terms of the Creative Commons Attribution 4.0 International license.

\begin{abstract}
Exercise is an essential aspect of our daily routine to maintain overall physical and mental health. Exercise and its influence on mental health is an emerging trend in the scientific research. Exercise not only improves physical health, but also influences long-term brain function and behaviour. In this review article, we have discussed the role of biological markers that reflect improvement in cognitive function in exercise. The understanding of how exercise ensures cognitive improvement is of paramount importance for implicating the role of exercise in reducing the burden of mental problems in the society.

Key words: Exercise, Cognition, P300, Reaction Time, Neurobiological evidence.
\end{abstract}

\section{INTRODUCTION}

Physical activity, due to its innumerable health benefits, is one of the oft-researched domains in medical literature. Apart from its health benefits, physical activity has a positive impact on cognition. ${ }^{[1]}$ Several human and animal studies have revealed the neurobiological mechanisms underlying the impact of physical activity on cognition. ${ }^{[2]}$ There has been a tremendous progress in the last decade about the molecular mechanisms, through which exercise improves mental health. The World Health Organization has suggested the daily recommendation of $60 \mathrm{~min}$ of moderate-to-vigorous intensity physical activity for children and adolescents (5 to 17 years) population. ${ }^{[1]}$

Regular or chronic exercise is a planned, structured and repetitive physical activity, which aims to improve and maintain physical fitness. Within the domain of physical activity, aerobic exercise seems to have more beneficial effects when compared to resistance exercises, though some of the studies have reported beneficial effects with both aerobic ${ }^{[3]}$ and resistance exercises. ${ }^{[4]}$

Aerobic exercises are defined as those that can stimulate the heart in order to increase the amount of oxygenated blood sent to working muscles and cells. ${ }^{[5]}$ Aerobic-based activities, including swimming, running, brisk walking and cycling, improve the oxygen transport to the body's cells and tissues. Aerobic exercise reduces the risk of cardiovascular disease, ${ }^{[6]}$ type 2 diabetes ${ }^{[7]}$ and specific types of cancers. ${ }^{[8]} \mathrm{Ad}-$ ditionally, aerobic exercise is associated with positive neurological and cognitive outcomes in children and older adults. ${ }^{[9]}$

In addition to the chronic regular exercise, the emerging researches indicates that even acute physical exercise has an obligatory role in improving alertness, processing speed and executive functions of cognition. ${ }^{[10]}$ While comparing low, moderate and high intensity exercises, studies have reported that cortical electric activity increases with increasing intensity of physical exercise, but reaches levels that favour cognitive performance at moderate intensities. $^{[11]}$

\section{Exercise and Cognition}

Exercise improves cognitive functioning and a wealthy of literature exists to support the notion. Exercise improves various components of cognitive function such as executive functions, selective attention, ${ }^{[12]}$ sustained attentional processes, ${ }^{[13]}$ concentration, reaction time, short term and long term memory and learning. ${ }^{[14]}$

\section{Chronic Exercise and Cognition}

A number of human and animal studies has supported the positive association between exercise on learning and memory. ${ }^{[15-19]} \mathrm{A}$ previous study has reported improved cognitive performance ${ }^{[20,21]}$ and declined neurodegeneration associated with aging ${ }^{[22]}$ in older adults. These effects are due to the increased hippocampal size with the exercise. In addition, children who engage in regular exercise performed better on verbal, perceptual, mathematical tests and overall academic performance ${ }^{[23]}$ when compared to children who do not engage in regular exercise or sports activities. Another study compared the Auditory and Visual Reaction Times (ART and VRT) of the individuals performing regular aerobic exercise and healthy adults. The study has reported a remarkable reduction in the ART and VRT in the group that performs regular aerobic exercise than the control group. These changes were independent of their age and gender. ${ }^{[24]}$

A number of experimental evidences are available for the impact of exercise on cognition. Animal studies in rodents have reported cognitive functioning 
enhancement with exercise. Running increases dendritic complexity and the number of dendritic spines in the dentate gyrus ${ }^{[25]}$ and entorhinal cortex.$^{[3]}$ Additional studies have reported an increase in the cell proliferation, neurogenesis, angiogenesis, dendritic complexity and spine density in rodents when they were subjected to exercise. ${ }^{[15]}$

In addition, studies also have reported improvement in learning and memory with exercise. This is because; exercise activates the transcriptional machinery inside the nucleus to modulate the expression of genes associated with regulation of synaptic plasticity, learning and memory using epigenetic mechanisms. ${ }^{[26]}$

\section{Acute Exercise and Cognition}

\section{Memory and Executive functions}

Emerging scientific researches reports that even acute exercise can bring about the beneficial effects equivalent to regular exercise both in terms of physical and mental health. Recently, a meta-analysis study reported that a single bout of moderate aerobic exercise improves inhibitory control, cognitive flexibility and working memory in preadolescent children and older adults. ${ }^{[27]}$ This documents the effect of acute exercise on cognition especially executive functions.

Studies have reported that acute bouts of physical exercise would benefit the cognitive performance in adults and elderly women ${ }^{[10]}$ and older adults. ${ }^{[28]}$ Another study has reported that acute exercise has helped to retain the information (working memory) for a longer period. ${ }^{[29]}$ A study in pre-schoolers proved that, involving in physical activity helped them to sustain the attention for a longer time, resulting in a better academic performance. ${ }^{[30]}$

Another study has reported improvement in sustained attention following acute bouts of both resistance and aerobic exercises, which was revealed by neurocognitive test batteries like Trail Making Test A and B (TMT-A and B). ${ }^{[31]}$ Exercise not only improves attention, it also improves motor memory and motor skill learning, concentration ${ }^{[32]}$ and enhances the working memory and executive functioning.

\section{Acute Exercise and Cognition - P300}

ERP has been useful in elucidating exercise-induced changes in processes occurring between stimulus engagement and response execution. As early as 1997, researchers have found that increasing amounts of physical exercise were related to increased amplitude of p300. The relationship between physical activity levels and executive functions were correlated with the results of p300 and Reaction Time (RT). A previous study has revealed that the physically active group fared better in the executive functions compared to the sedentary subjects. ${ }^{[33]}$ When p300 amplitude changes were investigated with various intensities of exercise, moderate intensity exercise demonstrated a greater enhancement with p300 amplitude than vigorous intensity exercise. ${ }^{[34]}$

Studies have reported an increase in p300 amplitude and decrease in p300 latency following exercise in preadolescent children, suggesting that acute exercise enhances executive aspects of cognition, which includes inhibition, working memory and cognitive flexibility. In addition, reduction in the reaction time and the P300 latency and improvement in the P300 amplitude has been recorded in pre-adolescent children, ${ }^{[35]}$ adults and in older population. ${ }^{[36-38]}$

Attention and academic performance increases with single acute bout of moderate-intensity exercise in adolescents, which was revealed by shortened P300 latencies. ${ }^{[9]}$ Another study elucidated the effects of acute physical exertion on Event Related Potentials (ERP). The researchers could observe significant changes in N100, P200, N200 and interpeak latencies. Females showed significant changes in N100, N200 and P200 latency. Both males and females exhibited a significant decrease in N200 latency for rare stimulus. ${ }^{[39]}$ When sedentary individuals were allowed to perform an acute bout of physical exercise, reduction in P300 latency was noted. ${ }^{[40]}$

A recent study has compared the effect of single acute bout of exercise on cognition in male athletic and non-athletic population. The study has reported a remarkable reduction in P300 latency, visual reaction time and auditory reaction time in both athletes and non-athletes following the exercise. This demonstrates the improvement in cognition with exercise irrespective of one being athlete or non-athlete. ${ }^{[41,42]}$ Therefore, it is evident that acute exercise improves cognitive functioning in all the agegroup individuals such as pre-adolescent children, adolescent, adults, elderly population irrespective of their past physical activity levels. During exercise, blood flow to the brain increases. In addition, neurogenesis and increased production of the neurotrophic factors like BDNF occurs. Exercise also modulates the molecular regulatory mechanisms involved in neurotransmission, metabolism and synaptic plasticity. ${ }^{[4-45]}$ All these mechanisms play crucial role in cognitive enhancement.

\section{Acute Exercise and Cognition - Reaction Time}

Improvement in cognitive functions can be assessed from the cognitive function tests like elucidating reaction time using the reaction time apparatus. There are two types of reaction time- visual and auditory reaction times. The mean auditory reaction time ART is comparatively shorter (284 ms) than mean visual reaction time VRT (331 ms). In addition, males were reported having faster ART and VRT compared to females. ${ }^{[46]}$ A recent study reported a remarkable reduction in the post-exercise reaction times in the individuals who were exposed to single acute bout of treadmill walking when compared to the baseline values. This is due to the exercise-induced improvement in the attentional scope in the individual. ${ }^{[47]}$ The authors further highlighted that acute exercise enhances the cognitive functioning by accentuating the efficacy of attentional system. ${ }^{[48]}$

To elucidate the effect of age on exercise-induced attentional control, a study compared the attentional span between 18 younger males and 17 older males using a go/no-go SART. The post-exercise reaction time was noted. Despite the age differences, both the groups displayed shorter reaction times after acute bout of exercise. ${ }^{[49]}$ Another study assessed the consequences of an acute bout of exercise on ART and VRT. They reported that an acute bout of cycling improved the auditory and visual reaction time parameters especially the ART. Therefore, it is evident that regular as well as acute bout of exercise dramatically improves the executive functions of cognition such as attention, concentration and reaction times of the individuals. ${ }^{[50]}$

\section{Exercise and Cognition: Neurobiological Evidence}

Recent studies have paid attention to beneficial effects of exercise on neurodegenerative disorders, which have resulted in newer insights. Exercise has been noted to enhance the neurogenesis of the hippocampal formation leading to better cognitive outcome ${ }^{[51]}$ in such individuals.

\section{Cortex and Hippocampus Involvement}

Exercise improves cognitive functioning by activating various areas of the cortex including the pre-frontal cortex.$^{[52]}$ Exercise stimulates the medial prefrontal cortex, which is associated with executive functions and working memory ${ }^{[53]}$ the striatum, associated with procedural memory; the hippocampus, associated with context-dependent learning and episodic memory. ${ }^{[54]}$

Studies have reported improved cognitive performance following a moderate intensity exercise, which was concurrently associated with left dorsolateral and prefrontal cortex activation. This provides evidence for the neural substrate involved in exercise-induced cognitive enhancement. [2] Another study attempted to evaluate the actions of acute exercise on frontal and medial temporal lobe-dependent cognitive functions. The study reported that high-intensity exercise had enhanced the cognitive 
functions pertaining to the prefrontal cortex. ${ }^{[5-56]}$ A recent study has found that acute exercise influences the prefrontal functions and cognitive functions by allele modification in Apo lipoprotein E $\varepsilon 4$ gene. They found a significant genotype-by-exercise interaction consequent to acute exercise. ${ }^{[57]}$

Animal studies in rodents have documented the involvement of neurobiological markers in the cognitive enhancement. Aerobic exercise increases the perfusion in the hippocampus in rodents, which could be mediated by exercise-induced angiogenesis. Animal studies suggest that angiogenesis is closely linked to adult neurogenesis. ${ }^{[8,59]}$ Runninginduced adult hippocampal neurogenesis substantially increases synaptic plasticity, spatial memory and pattern separation in adult animals. Exercise also inverses the declining neurogenesis and memory function in aged rodents. ${ }^{[60]}$

Therefore, it is evident from the above studies that there is a definite role and involvement of various lobes of the cortex and hippocampus in exercise associated enhancement in the cognition and in declining the ageassociated memory loss and neurodegeneration.

\section{Role of BDNF}

The benefit of exercise involves a number of biochemical markers. One among them is BDNF. BDNF is a neurotrophin, more commonly expressed in CNS, which has a critical role in hippocampal neurogenesis, neuroplasticity, neuronal shaping and survival. It is maximally produced in hippocampal and cortical neurons. BDNF is involved in the maturation and functioning of serotonin neurons. In turn, serotonin is involved in neuroplasticity phenomenon mediated by BDNF and stimulated by exercise. ${ }^{[61]}$ Aerobic exercise increases the BDNF concentration in most of the brain regions such as prefrontal cortex, peri-rhinal, striatum and hippocampus. These responses are independent of age factors. ${ }^{[62]} \mathrm{BDNF}$ and Serotonin converge, interact and mediate memory and learning processes.

A previous study evaluated the effect of acute exercise on serum BDNF levels. The study has reported that BDNF levels were greatly elevated following the exercise. The researchers also found that the level of rise in BDNF is directly proportional to the intensity of exercise. ${ }^{[63]}$ Recent studies have supported such observations suggesting a possible role of BDNF genotype in aerobic exercise-induced cortical brain differentiation. ${ }^{[64]}$

Certain animal studies have furthered our neurobiological understanding in the effects of exercise on cognitive functions. They have documented an increase in the NE receptor activation which further leads to increased cAMP-mediated signalling resulting in the proliferation of BDNF receptors. ${ }^{[65]}$

\section{Role of Serotonin}

Serotonin $(5-\mathrm{HT})$ is a neurotransmitter with a modulatory role in almost all functions and biological processes. The serotonin neurons and their projections innervate most brain areas, including cortical, limbic, midbrain, hindbrain and brainstem regions. Among the various receptors of serotonin, 5-HT1A receptor is involved in cognition. Therefore, it acts as a therapeutic target and a neural marker of memory deficits.

In a recent study, researchers elucidated the effects of varied intensities of exercise on serotonin levels. Young participants were randomly allocated to single acute bout of low intensity, moderate intensity and high intensity exercise. Compared to other groups, the high-intensity group had significantly higher values for serum serotonin, which indicates that levels of rise in serum serotonin are directly proportional to the intensity of the exercise. This study extends our observations in the field of exercise-induced physiological changes.

Animal studies have strengthened the above findings by reporting concomitant increase in the expression of serotonin and BDNF levels in the cortex and hippocampus with aerobic exercise. ${ }^{[6]}$ Serotonin also acts as a meta-modulator of other neurotransmitter systems involved in the formation of memory.

\section{CONCLUSION}

Exercise plays a crucial role in enhancing various components of cognition including executive functions like attention, concentration, reaction time ${ }^{[66,67]}$ and it has a remarkable role in modulating learning and longterm memory. Exercise brings about these beneficial changes by modulating various hormones, neurotrophins and neurotransmitters and by enhancing neurogenesis and synaptic plasticity. In addition, exercise is also vital for reducing the age-related memory deficits and neurodegeneration. With these existing data, it is clear that if more time is spent on exercise, especially aerobic activities; it may not only improve the physical health, but also positively influences the cognitive functioning of the individual.

\section{CONFLICT OF INTEREST}

The authors declare no conflict of interest.

\section{ABBREVIATIONS}

RT: Reaction Time; VRT: Visual Reaction Time; ART: Auditory Reaction Time; SART: Sustained Attention to Response Test; ERP: Event Related Potentials; BDNF: Brain-Derived Neurotrophic Factor.

\section{REFERENCES}

1. Herting MM, Chu X. Exercise, cognition and the adolescent brain. Birth Defects Research. 2017; 109(20):1672-9.

2. Yanagisawa H, Dan I, Tsuzuki D, Kato M, Okamoto M, Kyutoku Y, et al. Acute moderate exercise elicits increased dorsolateral prefrontal activation and improves cognitive performance with Stroop test. Neuroimage. 2010;50(4):1702 10.

3. Siette J, Reichelt AC, Westbrook RF. A bout of voluntary running enhances context conditioned fear, its extinction and its reconsolidation. Learning and Memory. 2014;21(2):73-81.

4. Strickland JC, Smith MA. Animal models of resistance exercise and their application to neuroscience research. Journal of Neuroscience Methods. 2016;273:191-200

5. Armstrong N, Welsman JR. Aerobic fitness: What are we measuring?. In: Pediatric Fitness. Karger Publishers. 2007;5-25.

6. Janssen I, LeBlanc AG. Systematic review of the health benefits of physical activity and fitness in school-aged children and youth. International Journal of Behavioral Nutrition and Physical Activity. 2010;7(1):40.

7. Stanford KI, Goodyear LJ. Exercise and type 2 diabetes: Molecular mechanisms regulating glucose uptake in skeletal muscle. Advances in Physiology Education. 2014;38(4):308-14

8. Ashcraft KA, Peace RM, Betof AS, Dewhirst MW, Jones LW. Efficacy and mechanisms of aerobic exercise on cancer initiation, progression and metastasis: A critical systematic review of in vivo preclinical data. Cancer Research. 2016;76(14):4032-50.

9. Hillman CH, Pontifex MB, Raine LB, Castelli DM, Hall EE, Kramer AF. The effect of acute treadmill walking on cognitive control and academic achievement in preadolescent children. Neuroscience. 2009;159(3):1044-54.

10. Córdova C, Silva VC, Moraes CF, Simões HG, DeNóbrega OT. Acute exercise performed close to the anaerobic threshold improves cognitive performance in elderly females. Brazilian Journal of Medical and Biological Research. 2009;42(5):458-64.

11. Chmura J, Nazar K, Kaciuba-Uścilko H. Choice reaction time during graded exercise in relation to blood lactate and plasma catecholamine thresholds. International Journal of Sports Medicine. 1994;15(04):172-6.

12. Bullock T, Giesbrecht B. Acute exercise and aerobic fitness influence selective attention during visual search. Frontiers in Psychology. 2014;5:1290.

13. Pontifex MB, Parks AC, Henning DA, Kamijo K. Single bouts of exercise selectively sustain attentional processes. Psychophysiology. 2015;52(5):618-25.

14. Sibley BA, Beilock SL. Exercise and working memory: An individual differences investigation. Journal of Sport and Exercise Psychology. 2007;29(6):783-91.

15. Lista I, Sorrentino G. Biological mechanisms of physical activity in preventing cognitive decline. Cellular and Molecular Neurobiology. 2010;30(4):493-503.

16. Cotman CW, Berchtold NC. Exercise: A behavioral intervention to enhance brain health and plasticity. Trends in Neurosciences. 2002;25(6):295-301.

17. Erickson KI, Voss MW, Prakash RS, Basak C, Szabo A, Chaddock L, et al. Exercise training increases size of hippocampus and improves memory. Proceedings of the National Academy of Sciences. 2011;108(7):3017-22. 
18. Praag VH, Kempermann G, Gage FH. Running increases cell proliferation and neurogenesis in the adult mouse dentate gyrus. Nature Neuroscience. 1999;2(3):266

19. Gomez-Pinilla F, Hillman C. The influence of exercise on cognitive abilities. Comprehensive Physiology. 2013;3(1):403-28

20. Colcombe SJ, Kramer AF, Erickson KI, Scalf P, McAuley E, Cohen NJ, et al. Cardiovascular fitness, cortical plasticity and aging. Proceedings of the National Academy of Sciences. 2004;101(9):3316-21.

21. Cassilhas RC, Viana VA, Grassmann V, Santos RT, Santos RF, Tufik S, et al. The impact of resistance exercise on the cognitive function of the elderly. Medicine and Science in Sports and Exercise. 2007;39(8):1401.

22. Smith GE, Housen P, Yaffe K, Ruff R, Kennison RF, Mahncke HW, et al. A cognitive training program based on principles of brain plasticity: Results from the Improvement in Memory with Plasticity-based Adaptive Cognitive Training (IMPACT) Study. Journal of the American Geriatrics Society. 2009;57(4):594-603.

23. Sibley BA, Etnier JL. The relationship between physical activity and cognition in children: A meta-analysis. Pediatric Exercise Science. 2003;15(3):243-56.

24. Garg M, Lata H, Walia L, Goyal O. Effect of aerobic exercise on auditory and visual reaction times: A prospective study. Indian J Physiol Pharmacol. 2013;57(2):138-45

25. Eadie BD, Redila VA, Christie BR. Voluntary exercise alters the cytoarchitecture of the adult dentate gyrus by increasing cellular proliferation, dendritic complexity and spine density. Journal of Comparative Neurology. 2005;486(1):39-47.

26. Fernandes J, Arida RM, Gomez-Pinilla F. Physical exercise as an epigenetic modulator of brain plasticity and cognition. Neuroscience and Biobehavioral Reviews. 2017;80:443-56

27. Ludyga S, Gerber M, Brand S, Holsboer-Trachsler E, Pühse U. Acute effects of moderate aerobic exercise on specific aspects of executive function in different age and fitness groups: A meta-analysis. Psychophysiology. 2016;53(11):161126.

28. Alves CR, Tessaro VH, Teixeira LA, Murakava K, Roschel H, Gualano B, et al. Influence of acute high-intensity aerobic interval exercise bout on selective attention and short-term memory tasks. Perceptual and Motor Skills. 2014;118(1):6372.

29. Coles K, Tomporowski PD. Effects of acute exercise on executive processing, short-term and long-term memory. Journal of Sports Sciences. 2008;26(3):33344.

30. Palmer KK, Miller MW, Robinson LE. Acute exercise enhances preschoolers' ability to sustain attention. Journal of Sport and Exercise Psychology. 2013;35(4):433-7.

31. Harveson AT, Hannon JC, Brusseau TA, Podlog L, Papadopoulos C, Durrant LH, et al. Acute effects of 30 min resistance and aerobic exercise on cognition in a high school sample. Research Quarterly for Exercise and Sport. 2016;87(2):21420.

32. Loprinzi PD, Nooe A. Executive function influences sedentary behavior: A longitudinal study. Health Promotion Perspectives. 2016;6(4):180.

33. Kamijo K, Takeda Y. Regular physical activity improves executive function during task switching in young adults. Int J Psychophysiol. 2010;75(3):304-11.

34. Kamijo K, Nishihira Y, Hatta A, Kaneda T, Wasaka T, Kida T, et al. Differential influences of exercise intensity on information processing in the central nervous system. European Journal of Applied Physiology. 2004;92(3):305-11.

35. Drollette ES, Scudder MR, Raine LB, Moore RD, Saliba BJ, Pontifex MB, et al. Acute exercise facilitates brain function and cognition in children who need it most: An ERP study of individual differences in inhibitory control capacity. Developmental Cognitive Neuroscience. 2014;7:53-64.

36. Kamijo K, HayashiY, Sakai T, Yahiro T, Tanaka K, Nishihira Y. Acute effects of aerobic exercise on cognitive function in older adults. J Gerontol B Psychol Sci Soc Sci. 2009;64(3):356-63.

37. Kamijo K, HayashiY, Sakai T, Yahiro T, Tanaka K, Nishihira Y. Acute effects of aerobic exercise on cognitive function in older adults. Journals of Gerontology: Series B. 2009;64(3):356-63.

38. Dai CT, Chang YK, Huang CJ, Hung TM. Exercise mode and executive function in older adults: An ERP study of task-switching. Brain and Cognition. 2013;83(2):153-62

39. Kumar N, Sood S, Singh M. Effect of acute moderate exercise on cognitive event-related potentials n100, p200, n200 and interpeak latencies. Indian Journal of Psychological Medicine. 2010;32(2):131.

40. Kumar N, Singh M, Sood S. Effect of acute moderate exercise on cognitive P300 in persons having sedentary lifestyles. International Journal of Applied and Basic Medical Research. 2012;2(1):67.

41. Kumar N, Singh M, Sood S, Beena N, Sakshi N, Roy PS, et al. Effect of acute moderate exercise on cognitive P300 in persons having sedentary lifestyles. In J Appl Basic Med Res. 2012;2(1):67-9.

42. Gowsi K, Sharma VK, Pal GK, Aaramban P. Effects of Moderate and High-Intensity Exercise on P300 Latency and Reaction Time in Athletes and NonAthletes-
An Interim Analysis. Biomedicine. 2016;36(4):60-5

43. Vaynman S, Gomez-Pinilla F. License to run: Exercise impacts functional plasticity in the intact and injured central nervous system by using neurotrophins. Neurorehabilitation and Neural Repair. 2005;19(4):283-95.

44. Cotman CW, Berchtold NC. Exercise: A behavioral intervention to enhance brain health and plasticity. Trends in Neurosciences. 2002;25(6):295-301.

45. Praag VH. Neurogenesis and exercise: Past and future directions. Neuromolecular Medicine. 2008;10(2):128-40.

46. Shelton J, Kumar GP. Comparison between auditory and visual simple reaction times. Neurosci Med. 2010;1(1):30-2.

47. Barnes RT, Coombes SA, Armstrong NB, Higgins TJ, Janelle CM. Evaluating attentional and affective changes following an acute exercise bout using a modified dot-probe protocol. J Sports Sci. 2010;28(10):1065-76.

48. Hogan M, Kiefer M, Kubesch S, Collins P, Kilmartin L, Brosnan M. The interactive effects of physical fitness and acute aerobic exercise on electrophysiological coherence and cognitive performance in adolescents. Experimental Brain Research. 2013;229(1):85-96.

49. Hsieh SS, Chang YK, Fang CL, Hung TM. Acute Resistance Exercise Facilitates Attention Control in Adult Males without an Age-Moderating Effect. Journal of Sport and Exercise Psychology. 2016;38(3):247-54.

50. Ashnagar Z, Shadmehr A, Jalaei S. The effects of acute bout of cycling on auditory and visual reaction times. Journal of Bodywork and Movement Therapies. 2015;19(2):268-72

51. Yau S, Gil-Mohapel J, Christie BR, So K. Physical exercise-induced adult neurogenesis: A good strategy to prevent cognitive decline in neurodegenerative diseases?. Bio Med Research International. 2014;2014

52. Badre $D$, Wagner AD. Left ventrolateral prefrontal cortex and the cognitive control of memory. Neuropsychologia. 2007;45(13):2883-901.

53. Brockett AT, LaMarca EA, Gould E. Physical exercise enhances cognitive flexibility as well as astrocytic and synaptic markers in the medial prefrontal cortex. PloS One. 2015;10(5):e0124859.

54. Shishmanova-Doseva M, Georgieva K, Koeva Y, Terzieva D, Peychev L. Enhancing effect of aerobic training on learning and memory performance in rats after long-term treatment with Lacosamide via BDNF-TrkB signaling pathway. Behavioural Brain Research. 2019;370:111963.

55. Yanagisawa H, Dan I, Tsuzuki D, Kato M, Okamoto M, Kyutoku Y, et al. Acute moderate exercise elicits increased dorsolateral prefrontal activation and improves cognitive performance with Stroop test. Neuroimage. 2010;50(4):170210.

56. Basso JC, Shang A, Elman M, Karmouta R, Suzuki WA. Acute exercise improves prefrontal cortex but not hippocampal function in healthy adults. Journal of the International Neuropsychological Society. 2015;21(10):791-801.

57. DeMarco M, Clough PJ, Dyer CE, Vince RV, Waby JS, Midgley AW, et al. Apolipoprotein E \& 4 Allele Modulates the Immediate Impact of Acute Exercise on Prefrontal Function. Behavior Genetics. 2015;45(1):106-16.

58. DeMarco M, Clough PJ, Dyer CE, Vince RV, Waby JS, Midgley AW, et al. Apolipoprotein E \&4 allele modulates the immediate impact of acute exercise on prefrontal function. Behav Genet. 2015;45(1):116-26.

59. JrLouissaint A, Rao S, Leventhal C, Goldman SA. Coordinated interaction of neurogenesis and angiogenesis in the adult songbird brain. Neuron 2002;34(6):945-60.

60. Opendak M, Gould E. Adult neurogenesis: A substrate for experience-dependent change. Trends in Cognitive Sciences. 2015;19(3):151-61.

61. Pietrelli A, Matković L, Vacotto M, Lopez-Costa JJ, Basso N, Brusco A. Aerobic exercise upregulates the BDNF-Serotonin systems and improves the cognitive function in rats. Neurobiology of Learning and Memory. 2018;155:528-42.

62. Baj G, D'alessandro V, Musazzi L, Mallei A, Sartori CR, Sciancalepore M, et al. Physical exercise and antidepressants enhance BDNF targeting in hippocampa CA3 dendrites: Further evidence of a spatial code for BDNF splice variants. Neuropsychopharmacology. 2012;37(7):1600.

63. Ferris LT, Williams JS, Shen CL. The effect of acute exercise on serum brainderived neurotrophic factor levels and cognitive function. Med Sci Sports Exerc. 2007;39(4):728-34.

64. Herting MM, Keenan MF, Nagel BJ. Aerobic fitness linked to cortical brain development in adolescent males: Preliminary findings suggest a possible role of BDNF genotype. Frontiers in Human Neuroscience. 2016;10:327.

65. McMorris T. Developing the catecholamines hypothesis for the acute exercisecognition interaction in humans: Lessons from animal studies. Physiology and behavior. 2016;165:291-99.

66. Duzel E, Praag VH, Sendtner M. Can physical exercise in old age improve memory and hippocampal function?. Brain. 2016;139(3):662-73

67. Gowsi K, Sharma VK, Pal GK, Aaramban P. An Experimental Study on the Effects of Acute Bouts of Exercise on Neurocognitive Performance in Athletes and Non-Athletes. EC Psychology and Psychiatry 7.9. 2018;597-603. 